Presented at the $6^{\text {th }}$ European Particle Accelerator Conference

Stockholm

June 22 to 261998

CONF-980671 -

\title{
RESULTS FROM A DOUBLE VLASOV MODEL FOR NEGATIVE ION EXTRACTION FROM VOLUME SOURCES
}

D. K. Olsen, R. J. Raridon, J. H. Whealton

Oak Ridge National Laboratory

Oak Ridge, Tennessee

\author{
Prepared by the \\ Oak Ridge National Laboratory \\ Oak Ridge, Tennessee 37831-8088 \\ Managed by \\ Lockheed Martin Energy Research Corporation \\ For the \\ U.S. Department of Energy \\ Under contract DE-AC05-960R22464
}

"The submitted manuscript has been authored by a contractor of the U.S. Government under contract DEAC05-96OR22464. Accordingly, the U.S. Government retains a nonexclusive, royalty-free license to publish or reproduce the published form of this contribution, or allow others to do so, for U. S. Government purposes."

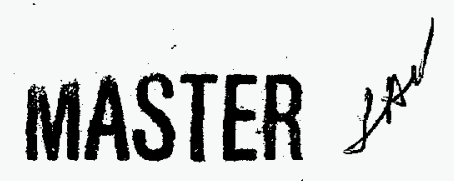

DISTRIBUTION OF THS DOCUMENT IS UNLMITED 


\section{DISCLAIMER}

This report was prepared as an account of work sponsored by an agency of the United States Government. Neither the United States Government nor any agency thereof, nor any of their employees, makes any warranty, express or implied, or assumes any legal liability or responsibility for the accuracy, completeness, or usefulness of any information, apparatus, product, or process disclosed, or represents that its use would not infringe privately owned rights. Reference herein to any specific commercial product, process, or service by trade name, trademark, manufacturer, or otherwise does not necessarily constitute or imply its endorsement, recommendation, or favoring by the United States Government or any agency thereof. The views and opinions of authors expressed herein do not necessarily state or reflect those of the United States Government or any agency thereof. 


\section{DISCLAIMER}

Portions of this document may be illegible electronic image products. Images are produced from the best available original document. 


\title{
RESULTS FROM A DOUBLE VLASOV MODEL FOR NEGATIVE ION EXTRACTION FROM VOLUME SOURCES
}

\author{
D. K. Olsen, R. J. Raridon, J. H. Whealton, ORNL, Oak Ridge, Tennessee
}

\section{Abstract}

A new negative ion source-extraction model has been formulated and implemented which explicitly considers the motion of positive ions and the volume generation of negative ions. It is found that: (1) for high-beam currents, the beam current is limited by a transverse spacecharge limit, not an emission limit; (2) there is a saddle point with a concomitant potential barrier preventing most volume produced negative ions from being extracted (the combination of 1 and 2 indicates that in some interesting cases there is the opportunity to increase extraction currents above values presently observed); (3) introduction of cesium may cause an actual increase in the transverse space-charge limit by accumulation of positive ions of cesium in the presheath.

\section{INTRODUCTION}

Intense negative ion beams of hydrogen or deuterium are of current interest in high-energy physics, nuclear physics and fusion technology. For example, pulsed spallation neutron sources are being proposed in both the United States (SNS) and Europe (ESS), which use charge exchange injection into a storage ring which in tum requires negative ion beams in the $\mathrm{GeV}$ range.

\section{I.I Negative ion extraction physics for volume sources}

For positive ion sources, the ionic flow is electrostatically attracted toward the extraction region and is either extracted or intercepted by the plasma electrode. Unfortunately, for negative ion sources these intraplasma electric fields tend to accelerate many, if not most, of the newly formed negative ions in the opposite direction or away from the extractor.

This and other issues require that the production of extractable negative ions needs to be understood in a selfconsistent manner. An accurate physics model for negative ion extraction is necessary, which is cognizant of some of the major asymmetries between negative ion extraction and positive ion extraction.

For positive ion extraction, the analysis is well developed through a self-consistent selection of equations (1) and (2) below:

$$
\begin{aligned}
& \nabla^{2} \varphi(r, t)=\int f(r, v, t) d v-\exp [-\varphi(r, t)] \\
& \frac{\partial f(r, v, t)}{\partial t}+v \bullet \nabla f(r, v, t)+\{v \times B-\nabla \varphi\} \bullet \nabla, f(r, v, t)=0
\end{aligned}
$$

The first asymmetry is that the equilibrium confined electrons cancel charge imbalance excursions in positive ion sources much better than the non-equilibrium, nonconfined positive ions do in negative ion sources. This observation led to the heuristic model, which was discussed previously. This heuristic model made some alterations to the plasma charge imbalance and initial data for the Vlasov equation. However, it is still common to model negative ion extraction from volume sources solving equations (1) and (2) by reversing the fields and charges without any further consideration of the positive ions, even in a heuristic way. We will call this the strict field reversal paradigm (SFRP). The SFRP has little meaning since it assumes that in negative ion sources positive ions are in confined equilibrium, which is not true and, furthermore, neglects an explicit consideration of plasma electrons. In addition, the SFRP tacitly assumes that the negative ions enjoy the same continuous acceleration from plasma to extraction that positive ions have in positive ion sources. This is also at variance with the experimental requirement of electrostatic plasma confinement.

The second asymmetry is the observation that the space potential is positive for negative ion sources just like in positive ion sources and for the same reason - to confine the plasma electrons electrostatically. This violates the SFRP that would have it that the space potential be negative. For negative ions, there is a saddle point with concomitant potential barrier formed within the plasma since the space potential lies intermediate between that of the plasma electrode and the acceleration electrode. The positive ions are first accelerated toward extraction until they reach the saddle ridge after which they decelerate, turn around, and either return to the plasma or intercept the plasma electrode. The negative ions are electrostatically repelled from the extractor in regions on 
the plasma side of the saddle ridge and are electrostatically attracted toward the extractor only in the region very near extraction region. Thus a large volume of negative ions are repelled from the extraction region. There is no extraction sheath since the plasma density is zero.

\subsection{Double-Vlasov model}

For volume negative ion sources it is necessary to consider the plasma positive ions kinetically as in Eq. 3; because they are far from equilibrium, executing at most two passes through the region (in a steady-state description). It is still appropriate to consider the electrons in the presheath to be a Boltzman distribution since they are collision dominated and generally have to traverse a magnetic field in the pre-extraction region. The model consists of a kinetic (Vlasov) equation for the positive ions, negative ions and electrons:

$$
\begin{aligned}
& \frac{\partial f_{+}}{\partial t}+v \bullet \nabla f_{+}+\{v \times B-\nabla \varphi\} \bullet \nabla_{v} f_{+}=\delta\left(S_{1}\right) \\
& \frac{\partial f_{-}}{\partial t}+v \bullet \nabla f_{-}-\{v \times B-\nabla \varphi\} \bullet \nabla_{v} f_{-}=G(r) \\
& \frac{\partial f_{e}}{\partial t}+v \bullet \nabla f_{e}-\frac{M}{m_{e}}\{v \times B-\nabla \varphi\} \bullet \nabla_{v} f_{e}=\delta\left(S_{2}\right) \\
& \nabla^{2} \varphi=\int\left\{f_{+}(v)-f_{-}(v)-f_{e}(v)\right\} d v-e^{-\varphi}
\end{aligned}
$$

Equations (3)-(6) are coupled to a Poisson Equation where the charge density of the above species is considered along with an equilibrium Boltzman density for the presheath electrons

A case with significant plasma density and dimensions equal to a thousand Debye lengths is shown in Fig. 1a. Positive ions are accelerated from the center of the plasma, as shown in Fig. 1, until they reach the saddle ridge. Then the positive ions are repelled by the accelerator fields and are attracted into the plasma electrode as shown. This is in contrast to positive ions. For volume produced negative ions, the trajectories are shown in Fig. Ib. Here, only a fraction of the negative ions produced are extracted, and the rest are attracted to the center of the plasma since the plasma electrode repulses them.

\subsection{Transverse Space-Charge Limits}

When the extracted current depends on extraction voltage, which is usually the case at high currents, the beam is said to be transverse space charge limited (TSCL). This is because the extraction voltage has very little effect on the negative ion generation rate, and so the beam is limited by interception on one or more electrodes. This interception comes about by poor optics due to an over supply of space charge. The very nature of a transverse space-charge limit means, if the generation rate is increased (beyond any decrease in electron space charge), the beam current actually decreases.

\subsection{Increasing the Transverse Space-Charge Limit (TSCL)}

It can be shown that cesium allows the negative ions to occupy virtually the entire transverse space-charge limit, it remains to examine if and how the space-charge limit can itself be increased. The transverse space-charge limit is usually thought to depend on the geometry of the electrodes, potentials applied, and beam current density. Actually, the transverse space-charge limit depends also on the shape of the extraction sheath, which is normally determined by the above-mentioned parameters, but by artificial means can be further manipulated. For example, the deliberate introduction of an additional positive charge into the pre-extraction-sheath region would have the effect of changing the curvature of the sheath affecting a higher space-charge limit. This is illustrated in Fig. 2, which represents a self-consistent solution to Eqs. (3). (4), and (6). Ionized sputtered cesium is just such a source of positive ions. The transverse space-charge limit is increased by an extra supply of positive charge into the presheath. This can occur because positive ions hitting the plasma electrode can sputter off neutral cesium, which quickly gets ionized by electrons and $\mathrm{H}^{+}$after passing through the sheath between the source plasma and the plasma electrode. These $\mathrm{Cs}^{+}$ions are heavy and slow and so are quite effective in depositing positive space charge into the presheath. The $\mathrm{Cs}^{+}$space charge in the region, just before extraction, serves to increase the transverse spacecharge limit by causing the extraction sheath to be more concave (Fig. 2 compared with Fig. 1).

\section{SUMMARY}

In summary, a new model has been described that takes into account several new features of negative ion extraction. The new model gives insight into such important observations as increased beam current when 
cesium is added to negative ion volume sources and an increase in the transverse space-charge limit itself.

\section{FIGURE LEGENDS}

Fig. 1a. Solution to Eqs. (3), (4) and (6) explicitly showing the positive ion distribution (solid lines) and equipotentials (dashed lines).

Fig. 1b. Solution to Eqs. (3), (4) and (6) explicitly showing the negative ion distribution (solid lines) and equipotentials (dashed lines).

Fig. 2a. Same as Fig. 1a but with extra positive charge in the presheath plasma.

Fig. 2b. Same as Fig. lb but with extra negative charge in the presheath plasma. 


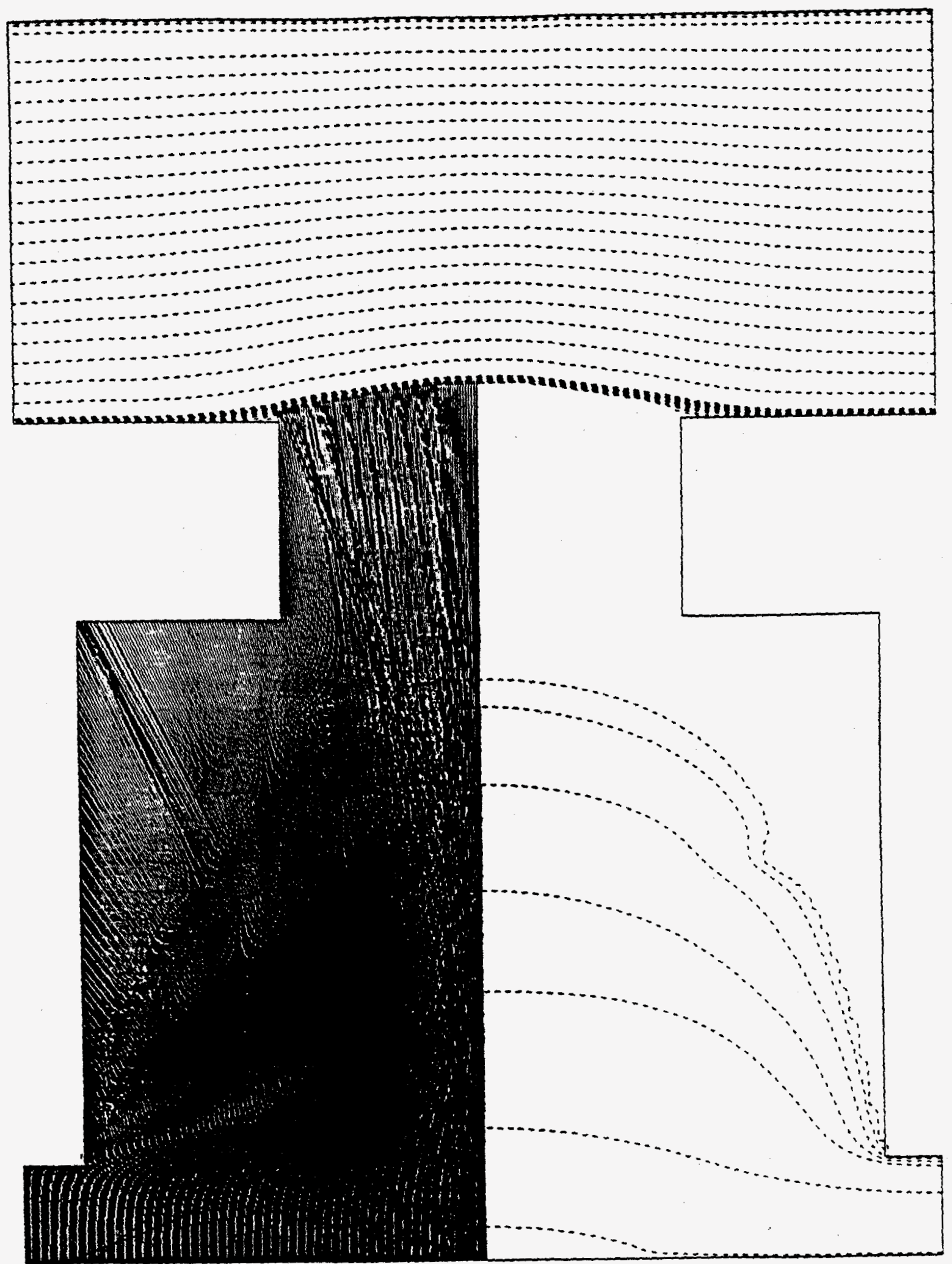

Fig. 1a. Solution to Eqs. (3), (4) and (6) explicitly showing the positive ion distribution (solid lines) and equipotentials (dashed lines). 


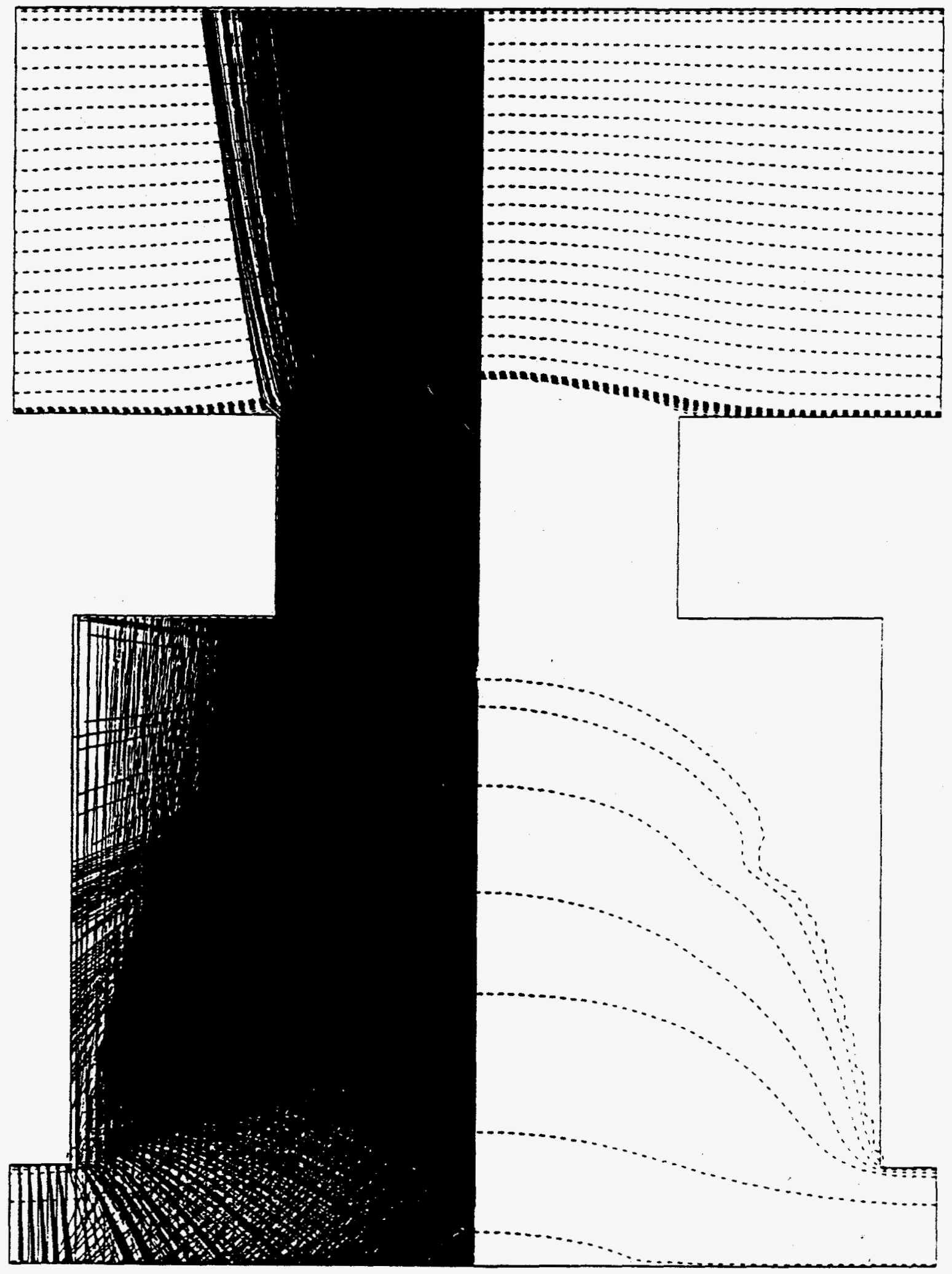

Fig. 1b. Solution to Eqs. (3), (4) and (6) explicitly showing the negative ion distribution (solid lines) and equipotentials (dashed lines). 


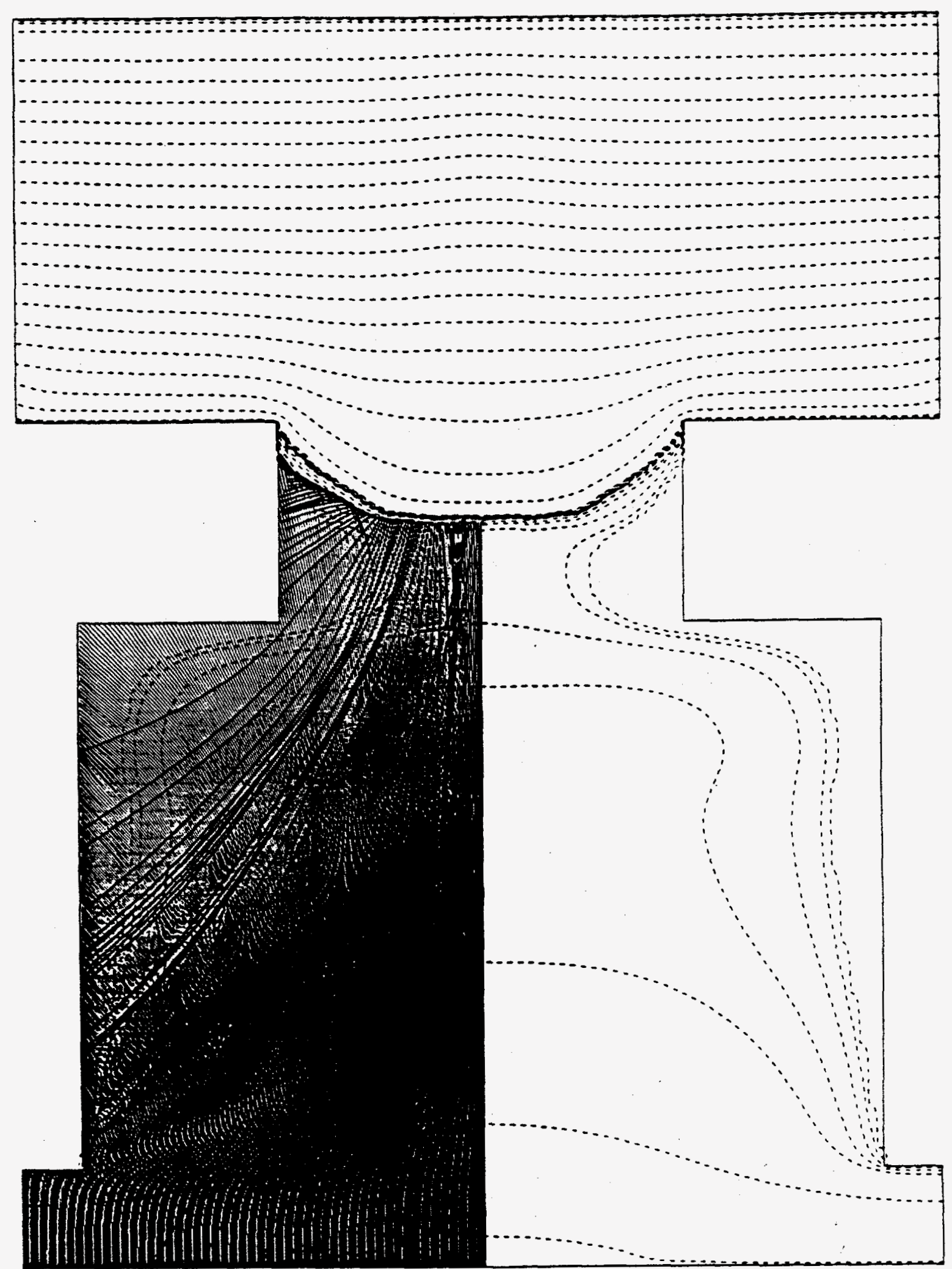

Fig. 2a. Same as Fig. 1a but with extra positive charge in the presheath plasma. 


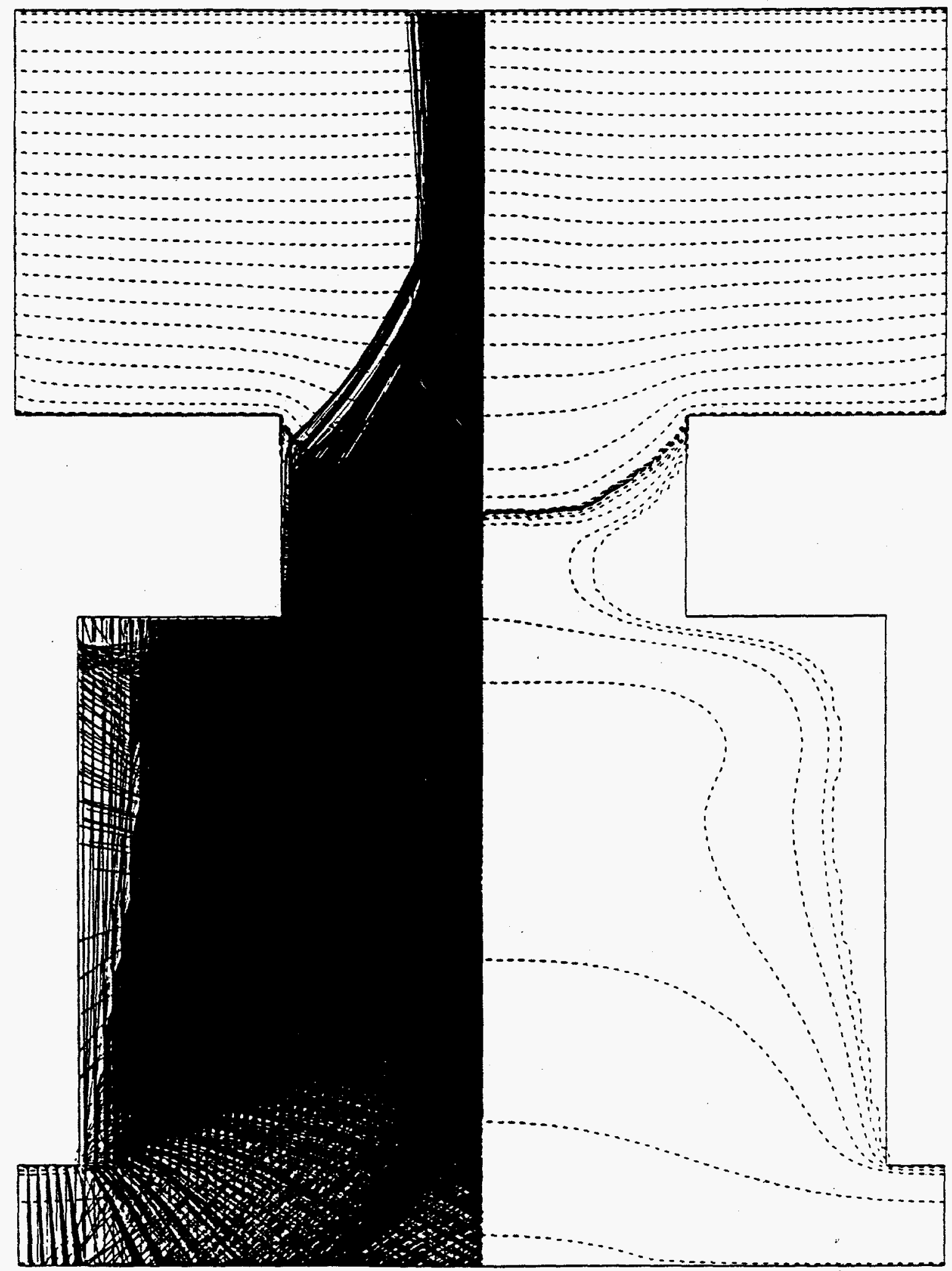

Fig. 2b. Same as Fig. $1 \mathrm{~b}$ but with extra negative charge in the presheath plasma. 